\title{
Candidate quality in a Downsian Model with a Continuous Policy Space. ${ }^{1}$
}

\author{
Enriqueta Aragonès ${ }^{2}$ \\ Institut d'Anàlisi Econòmica, CSIC
}

\author{
Dimitrios Xefteris ${ }^{3}$ \\ University of Cyprus
}

January 10, 2011

\footnotetext{
${ }^{1}$ Aragones acknowledges financial support by the Generalitat de Catalunya Grant number 2009 SGR 1126, the Spanish Ministry of Education grant number ECO2009-08820 and the Barcelona Graduate School of Economics.

${ }^{2}$ Institut d'Anàlisi Econòmica, CSIC, Campus UAB, 08193 Bellaterra (Spain). Email: enriqueta.aragones@iaea.csic.es

${ }^{3}$ Department of Economics, Faculty of Economics and Management, University of Cyprus. P.O. Box 20537, CY-1678 Nicosia (Cyprus). Email: xefteris.dimitrios@ucy.ac.cy
} 


\begin{abstract}
This paper characterizes a mixed strategy Nash equilibrium in a one-dimensional Downsian model of two-candidate elections with a continuous policy space, where candidates are office motivated and one candidate enjoys a nonpolicy advantage over the other candidate. We assume that voters have quadratic preferences over policies and that their ideal points are drawn from a uniform distribution over the unit interval. In our equilibrium the advantaged candidate chooses the expected median voter with probability one and the disadvantaged candidate uses a mixed strategy that is symmetric around it. We show that this equilibrium exists if the number of voters is large enough relative to the size of the advantage.
\end{abstract}

Key words: spatial competition; mixed strategies; candidate quality 


\section{Introduction}

Candidate quality is considered to be a critical variable in electoral competition. It affects the decisions of politicians regarding whether to run for office, campaign fund-raising, voter behavior, election outcomes, and, ultimately, policy outcomes. Quality differences between two candidates can arise for many reasons, including charisma, office-holding experience, incumbency, advertising, scandal, and any other non-policy dimension that may affect the relative attractiveness of two candidates. In Political Science candidate quality is also denoted by "valence dimension" and its importance has been widely demonstrated over several decades of careful empirical research. ${ }^{1}$

All else constant, high quality candidates will fare better than low quality candidates. Furthermore, quality differences produce significant changes in the nature of political competition. The equilibrium properties of spatial competition between two candidates who differ in quality have been analyzed theoretically. Recent papers by Ansolabehere and Snyder (2000), Aragones and Palfrey (2002), Groseclose (2001) and Hummel (2010) report a number of theoretical results about the equilibrium properties of spatial competition between two candidates who differ in quality. ${ }^{2}$

These papers use a framework for studying the effect of candidate quality on political competition, based on the standard Downsian model competition between two candidates with an important twist: any voter will strictly prefer the "higher quality" candidate to the "lower quality" candidate if the candidates locate so that the voter is indifferent between the two candidates on the policy dimension.

Groseclose (2001) shows that, for office motivated candidates, existence of pure strategy equilibrium is especially problematic for small-to-intermediate values of the quality advantage. When the policy space is an interval of the real line and voters' preferences are Euclidean the payoff functions of the candidates are discontinuous. This discontinuity implies that the best response of the disadvantaged candidate is not well defined. In this set up Aragones and Palfrey (2002) described the equilibrium strategies for a particular case: when the policy space is a finite grid of

\footnotetext{
${ }^{1}$ See, e.g., Stokes (1963), Kiewiet (1983), and Kiewiet and Zheng (1993).

${ }^{2}$ There are also some earlier theoretical papers that studied related kinds of asymmetry, such as incumbency or partisanship, e.g., Adams (1998), Bernhardt and Ingberman (1985), and Londregan and Romer (1993).
} 
points on the $[0,1]$ interval and when the advantage is relatively small. Hummel (2010) studies the same environment with the only difference that he considers that the advantage might be higher than Aragones and Palfrey (2002). In this case the median voter might prefer to vote for the candidate with superior valence even if he prefers the policy of the other candidate. Hummel (2010) characterizes the optimal actions of both candidates in a particular equilibrium of the game but, unlike Aragones and Palfrey (2002), he does not fully characterize any equilibrium of the game.

The characterization of the equilibrium strategies for a continuous policy space has not been studied so far and it is the main objective of this paper. The model we analyze follows the standard Downsian model with a continuous policy space and the following modifications: we assume that voters have quadratic preferences instead of Euclidean and we assume that the beliefs of candidates' on the distribution of the median voter's ideal point are unimodal (not necessarily uniform). By changing the voters' preferences from linear to quadratic the payoff functions of the candidates become continuous and the best response of the disadvantaged candidate is well defined. Within this set up we are able to find conditions for existence of mixed strategy equilibrium and we are also able to describe the equilibrium strategies.

We find a family of unimodal distributions for the median voter's ideal point that guarantees existence of an equilibrium in which the advantage candidate chooses a pure strategy that concentrates all the probability in the expected location of the median voter, while the disadvantaged candidate chooses a mixed strategy that allocates probability symmetrically around the expected location of the median voter.

We find necessary and sufficient conditions for this equilibrium to exist. These conditions impose restrictions only on the candidates' beliefs on the voters' distribution of preferences. We find that this equilibrium exists only if the level of uncertainty about the location of the median voter is low enough relative to the size of the advantage, that is, when candidates believe that the median voter's ideal point is around $1 / 2$ with high enough probability.

The conditions we find for existence do not restrict at all the size of the quality advantage, that is, the difference in quality between the two candidates, as it was the case in Groseclose (2001). 
Thus we show that this mixed strategy equilibrium may exist even for small values of the quality advantage, which is when pure strategy equilibrium fails to exist.

As in similar models, we find that in equilibrium the advantaged candidate obtains a larger probability of winning than the disadvantaged candidate. We also find that as the value of the advantage becomes larger the probability of wining of the advantaged candidate increases, the equilibrium strategies of the two candidates are more differentiated, and the conditions of existence of equilibrium are relaxed, that is, the number of voters (or the probability with which the expected median voter is around 1/2) needed for an equilibrium to exist is smaller. Finally, as the value of the advantage becomes smaller, that is, as the difference between the two candidates vanishes, the optimal strategy of the disadvantaged candidate moves closer to the advantage candidate's. That is both players' equilibrium strategies converge to the expected median voter as candidate A's advantage shrinks to zero.

The rest of the paper proceeds as follows. The next section describes the formal model. Section 3 presents the derivation of the equilibrium strategies and analyzes its properties. Finally, section 4 contains some concluding remarks.

\section{The Model}

The policy space is the $[0,1]$ interval. There are $n$ voters, where $n$ is an odd and finite number. Each voter has a utility function, with two components, a policy component, and a candidate image component. The policy component is characterized by an ideal point in the policy space, with utility of alternatives in the policy space a quadratic function of the distance between the ideal point and the location of the policy. The image component is captured by an additive constant to the utility a voter gets if the higher quality candidate wins the election.

There are two candidates, $A$ and $D$, who are referred to as the advantaged candidate and the disadvantaged candidate, respectively. Each candidate's objective is to maximize his probability of winning the election. We assume that candidates believe that the ideal point of each voter is 
an i.i.d. draw from a uniform distribution in $[0,1]$.

The game takes place in two stages. In the first stage, candidates simultaneously choose positions in $[0,1]$. In the second stage, each voter votes for the candidate whose election would give him the highest utility. In case of indifference, a voter is assumed to vote for each candidate with probability equal to $1 / 2$.

Let $x$ denote the policy position chosen by candidate $A$, and let $y$ denote the policy position chosen by candidate $D$. Then, the utility that a voter with ideal point $x_{i}$ obtains if $A$ wins the election is given by $U_{i}(x)=d-\left(x_{i}-x\right)^{2}$ and his utility if candidate $D$ wins is given by $U_{i}(y)=$ $-\left(x_{i}-y\right)^{2}$, where $d>0$ denotes the size of candidate $A$ 's advantage.

Since the behavior of the voters is unambiguous in this model, we define an equilibrium of the game only in terms of the location strategies of the two candidates in the first round. A pure strategy equilibrium is a pair of candidate locations $(x, y)$ such that both candidates are maximizing the probability of winning, given the choices of the other candidate. A mixed strategy equilibrium is a pair of probability distributions $\left(\sigma^{A}, \sigma^{D}\right)$ over $[0,1]$ such that there is no mixed strategy for $A$ that guarantees higher probability of winning than $\sigma^{A}$, given $\sigma^{D}$, and there is no mixed strategy for $D$ that guarantees higher probability of winning than $\sigma^{D}$, given $\sigma^{A}$.

Notice that in this set up, if $x<y$ then all voters with $d-\left(x_{i}-x\right)^{2}>-\left(x_{i}-y\right)$ prefer to vote for candidate $A$. Therefore, we have that all voters with an ideal point $x_{i}<\frac{x+y}{2}+\frac{d}{2(y-x)}=\widehat{x}(x, y)$ prefer to vote for candidate $A$. Since the ideal point of each voters is drawn from a uniform distribution, this implies that the probability that a voter votes for the advantaged candidate is given by $p(x, y)=\min \{\widehat{x}(x, y), 1\}$ and the probability that a voter votes for the disadvantaged candidate is given by $q(x, y)=\max \{0,1-\widehat{x}(x, y)\}$.

Similarly if $x>y$ we have that all voters with an ideal point $x_{i}>\frac{x+y}{2}+\frac{d}{2(y-x)}=\widehat{x}(x, y)$ prefer to vote for candidate $A$. This implies that the probability that a voter votes for the advantaged candidate is given by $p(x, y)=\min \{1,1-\widehat{x}(x, y)\}$ and the probability that a voter votes for the disadvantaged candidate is given by $q(x, y)=\max \{0, \widehat{x}(x, y)\}$. 
Since we assume that there are $n$ voters, the probability with which the advantaged candidate wins the election is given by the probability that the advantaged candidate obtains the votes of at least a majority of the voters. Because each voter will vote for candidate $A$ with probability $p(x, y)$ the probability with which candidate $A$ is elected may be computed by the sum of the Bernoulli distributions corresponding to at least a majority of successes over $n$ trials, that is,

$$
P_{n}(x, y)=\sum_{k=\frac{n+1}{2}}^{n}\left(\begin{array}{l}
n \\
k
\end{array}\right) p(x, y)^{k}(1-p(x, y))^{n-k}
$$

Similarly we could also show that the probability with which the disadvantage candidate wins the election is given by $Q_{n}(x, y)=\sum_{k=\frac{n+1}{2}}^{n}\left(\begin{array}{l}n \\ k\end{array}\right) q(x, y)^{k}(1-q(x, y))^{n-k}=\sum_{k=\frac{n+1}{2}}^{n}\left(\begin{array}{l}n \\ k\end{array}\right)(1-p(x, y))^{k} p(x, y)^{n-k}=$ $1-P_{n}(x, y)$

Observe that $p(x, y)$ and $q(x, y)$ are continuous functions of $x \in[0,1]$ and $y \in[0,1]$, and therefore $P_{n}(x, y)$ and $Q_{n}(x, y)$ are continuous functions of $x \in[0,1]$ and $y \in[0,1]$ as well.

Finally, if $x=y$, we have that $p(x, y)=1$ and $q(x, y)=0$, that is, if both candidates choose the same location then the advantaged candidate wins with probability one, because in this case all voters would prefer to vote for him.

Notice that the payoff functions of the candidates in our set up coincide with the c.d.f. of a Beta distribution with parameters $a=b=\frac{n+1}{2}$. Such a distribution is unimodal and symmetric around $\frac{1}{2}$. This observation allows us to offer an alternative interpretation of our model. Suppose that we have any number of voters, even a continuum, and a unique median voter. Suppose that the candidates' beliefs about the distribution of the median voter's ideal point are represented by this Beta distribution. In this case the candidates' payoff functions would be represented by the same family of c.d.f. parametrized by the parameter of the Beta distribution instead of the number of voters of the original set up. In this case $p(x, y)$ would represent the ideal point of the voter that is indifferent between the two candidates. Thus in both cases we have that the candidates' beliefs about the location of the median voter's ideal point are more concentrated around $\frac{1}{2}$ whenever the number of voters increases or, what is the same, when the parameter $a$ of the Beta distribution increases. 
The payoff functions of the candidates in our set up also coincide with those of the Condorcet jury members (see, for example, Kirstein and Wangenheim, 2010). This coincidence will prove to be helpful for our analysis.

\section{Equilibrium Strategies}

When $d=0$, neither candidate has an advantage, and we are in the standard Downsian world, where in equilibrium the two candidates locate at $\frac{1}{2}$ and each wins with probability $\frac{1}{2}$. In general, when $d>0$, there does not exist a pure strategy equilibrium. Different versions of this result have been stated and proven in Groseclose (1999) and Berger, Munger, and Potthoff (1999). The intuition is simple. If the disadvantaged candidate's location is perfectly predictable, the advantaged candidate can copy that strategy and win for sure. Therefore, at least the disadvantaged candidate must be mixing. The result is true unless $d$ is sufficiently large that $A$ can locate at the median and guarantee a payoff of 1 .

Proposition 1 If $d \geq \frac{1}{4}$ there is a pure strategy equilibrium in which $A$ wins with probability one.

(All proofs may be found in the appendix.)

In our case, if $d<\frac{1}{4}$, then there will be no pure strategy equilibrium. The aim of this paper is to show that if $d \in\left(0, \frac{1}{4}\right)$ there exists a mixed strategy Nash equilibrium ${ }^{3}$ in which the advantaged candidate chooses a pure strategy and the disadvantaged candidate chooses a mixed strategy. In particular we show that in this equilibrium the advantaged candidate chooses a pure strategy corresponding to the ideal point of the expected median voter, $x=\frac{1}{2}$, while the disadvantaged candidate mixes between the two policy locations $y=\frac{1}{2}-\sqrt{d}$ and $y=\frac{1}{2}+\sqrt{d}$ each with equal probability. We find that this equilibrium exists as long as the number of voters is large enough relative to the size of the advantage $d$. We also find the minimal number of voters that guarantees

\footnotetext{
${ }^{3}$ If candidates have private information with continuous types, then this mixed equilibrium can be "purified." That is, there will exist an equilibrium in pure strategies, where the equilibrium locations of candidates will vary with this private information (Aragones and Palfrey 2005).
} 
existence of this Nash equilibrium as a function of the size of the advantage.

We start by demonstrating that the strategy proposed for candidate $D, \widetilde{\sigma}^{D}=\left(y=\frac{1}{2}-\sqrt{d}\right.$ with probability $\frac{1}{2}$ and $y=\frac{1}{2}+\sqrt{d}$ with probability $\frac{1}{2}$ ) is an optimal response to candidate $A$ choosing $\widetilde{\sigma}^{A}=\frac{1}{2}$. We prove that this holds true for all values of $n$.

Proposition 2 For all $n>0$ and for all $0<d<\frac{1}{4}$ we have that $\widetilde{\sigma}^{D}=\left(y=\frac{1}{2}-\sqrt{d}\right.$ with probability $50 \%$ and $y=\frac{1}{2}+\sqrt{d}$ with probability 50\%) is a best response to $\tilde{\sigma}^{A}=\frac{1}{2}$.

Next we have to show that the strategy proposed for candidate $A, \widetilde{\sigma}^{A}=\frac{1}{2}$, is a best response to candidate $D$ choosing $\widetilde{\sigma}^{D}=\left(y=\frac{1}{2}-\sqrt{d}\right.$ with probability $\frac{1}{2}$ and $y=\frac{1}{2}+\sqrt{d}$ with probability $\left.\frac{1}{2}\right)$. Notice that when candidate $D$ is choosing strategy $\widetilde{\sigma}^{D}=\left(\frac{1}{2}-\sqrt{d}\right.$ w.p. $\frac{1}{2} ; \frac{1}{2}+\sqrt{d}$ w.p. $\left.\frac{1}{2}\right)$ candidate $A$ 's probability of election is given by:

$$
P_{n}\left(x, \widetilde{\sigma}^{D}\right)=\frac{1}{2} P_{n}(x, 1 / 2-\sqrt{d})+\frac{1}{2} P_{n}(x, 1 / 2+\sqrt{d})
$$

where

$$
P_{n}(x, 1 / 2-\sqrt{d})=\sum_{k=\frac{n+1}{2}}^{n}\left(\begin{array}{l}
n \\
k
\end{array}\right) p(x, 1 / 2-\sqrt{d})^{k}(1-p(x, 1 / 2-\sqrt{d}))^{n-k}
$$

and

$$
P_{n}(x, 1 / 2+\sqrt{d})=\sum_{k=\frac{n+1}{2}}^{n}\left(\begin{array}{l}
n \\
k
\end{array}\right) p(x, 1 / 2+\sqrt{d})^{k}(1-p(x, 1 / 2+\sqrt{d}))^{n-k}
$$

Moreover, observe that the function $p(x, y)$ increases with $x$ for all $x<1-\sqrt{d-2 y+y^{2}+1}$, remains constant $(p(x, y)=1)$ for $x \in\left[1-\sqrt{d-2 y+y^{2}+1}, \sqrt{y^{2}+d}\right]$ and decreases with $x$ for all $x>\sqrt{y^{2}+d}$.

[Figure 1]

That is, $p(x, 1 / 2-\sqrt{d})$ is increasing in $x \in\left[0,1-\sqrt{\frac{1}{4}+2 d+\sqrt{d}}\right)$, constant in $x \in[1-$ $\left.\sqrt{\frac{1}{4}+2 d+\sqrt{d}}, \sqrt{\frac{1}{4}+2 d-\sqrt{d}}\right]$ and decreasing in $x \in\left(\sqrt{\frac{1}{4}+2 d-\sqrt{d}}, 1\right]$ and $p(x, 1 / 2+\sqrt{d})$ is increasing in $x \in\left[0,1-\sqrt{\frac{1}{4}+2 d-\sqrt{d}}\right)$, constant in $x \in\left[1-\sqrt{\frac{1}{4}+2 d-\sqrt{d}}, \sqrt{\frac{1}{4}+2 d+\sqrt{d}}\right]$ and 
decreasing in $x \in\left(\sqrt{\frac{1}{4}+2 d+\sqrt{d}}, 1\right]$. Since $\sqrt{\frac{1}{4}+2 d-\sqrt{d}}<\sqrt{\frac{1}{4}+2 d+\sqrt{d}}$ for any $d \in\left(0, \frac{1}{4}\right)$ and since $\frac{\partial P_{n}(x, y)}{\partial p}>0$, it naturally follows that $P_{n}\left(x, \widetilde{\sigma}^{D}\right)=\frac{1}{2} P_{n}(x, 1 / 2-\sqrt{d})+\frac{1}{2} P_{n}(x, 1 / 2+\sqrt{d})$ is increasing in $x \in\left[0, \sqrt{\frac{1}{4}+2 d-\sqrt{d}}\right)$ and that $P_{n}\left(x, \widetilde{\sigma}^{D}\right)$ is decreasing in $x \in\left(1-\sqrt{\frac{1}{4}+2 d-\sqrt{d}}, 1\right]$ for any $n>0$.

This observation is very helpful because it implies that when $D$ chooses the proposed mixed strategy $\widetilde{\sigma}^{D}$ the best response of $A$ must belong in $\left[\sqrt{\frac{1}{4}+2 d-\sqrt{d}}, 1-\sqrt{\frac{1}{4}+2 d-\sqrt{d}}\right]$ for any $n>0$.

We first analyze the case of a single voter, that is, $n=1$, which corresponds to an expected median voter's ideal point drawn from a uniform distribution on $[0,1]$. In this case, we find that the proposed strategies do not form an equilibrium. However this result will help us to illustrate the path that we will follow to demonstrate the main result. Since we know that the proposed strategy for candidate $D$ is a best response for all values of $n$, then it must be the case that the proposed strategy for candidate $A$ is not.

Proposition 3 If $n=1$ and $0<d<\frac{1}{4}$ then $x=\frac{1}{2}$ is not a best response for $A$ to $\widetilde{\sigma}^{D}$.

The specific shape of the payoff function of the advantaged candidate for the case $n=1$ illustrates the reason why $x=\frac{1}{2}$ is not a best response. The payoff function is convex around $\frac{1}{2}$ and thus the function $P_{n}\left(x, \widetilde{\sigma}^{D}\right)$ is achieves a local minimum at $x=\frac{1}{2}$. This will still be the case for small values of $n$. But as $n$ increases the values of the payoff function around $\frac{1}{2}$ will increase and the function will become concave exhibiting a global maximum at $\frac{1}{2}$ when $n$ is large enough (See figures 3, 4 and 5.).

In order to prove that $\widetilde{\sigma}^{A}=1 / 2$ is a global maximum of $P_{n}\left(x, \widetilde{\sigma}^{D}\right)$ we have to show that $P_{n}\left(x, \tilde{\sigma}^{D}\right)$ is increasing for all $x \in[0,1 / 2]$ and decreasing for all $x \in[1 / 2,1]$. Remember that when $x \in[0, \sqrt{1 / 4+2 d-\sqrt{d}}]$ we have that both $p(x, 1 / 2-\sqrt{d})$ and $p(x, 1 / 2+\sqrt{d})$ are increasing functions of $x$, thus $P_{n}\left(x, \widetilde{\sigma}^{D}\right)$ is increasing for all $n$ in this range. Similarly, when $x \in[1-$ $\sqrt{1 / 4+2 d-\sqrt{d}}, 1]$ we have that both $p(x, 1 / 2-\sqrt{d})$ and $p(x, 1 / 2+\sqrt{d})$ are decreasing functions of $x$, thus $P_{n}\left(x, \widetilde{\sigma}^{D}\right)$ is increasing for all $n$ in this range. 
Next, we show that as $n$ increases the slope of $P_{n}\left(x, \widetilde{\sigma}^{D}\right)$ will gradually become positive for $x \in[\sqrt{1 / 4+2 d-\sqrt{d}}, 1 / 2)$ and negative for $x \in(1 / 2,1-\sqrt{1 / 4+2 d-\sqrt{d}}]$, while the sign of the slope everywhere else does not change, which will lead to the conclusion that $\widetilde{\sigma}^{A}=1 / 2$ is indeed the unique best response of $A$ to $\widetilde{\sigma}^{D}$ for some large $n$.

We call $x \in(\sqrt{1 / 4+2 d-\sqrt{d}}, 1-\sqrt{1 / 4+2 d-\sqrt{d}})$ the critical values of the payoff function of the advantaged candidate (see figure 6). The next proposition shows that for $n$ large enough $\frac{\partial P_{n}\left(x, \widetilde{\sigma}^{D}\right)}{x}>0$ for $x \in\left[\sqrt{1 / 4+2 d-\sqrt{d}}, \frac{1}{2}\right)$ and $\frac{\partial P_{n}\left(x, \widetilde{\sigma}^{D}\right)}{x}<0$ for $x \in\left(\frac{1}{2}, 1-\sqrt{1 / 4+2 d-\sqrt{d}}\right]$ which should be enough to conclude that $\widetilde{\sigma}^{A}=1 / 2$ is indeed a global maximum of $\partial P_{n}\left(x, \widetilde{\sigma}^{D}\right)$.

Proposition 4 For $n$ large enough we have that $\frac{\partial P_{n}\left(x, \widetilde{\sigma}^{D}\right)}{x}>0$ for $x \in\left[\sqrt{1 / 4+2 d-\sqrt{d}}, \frac{1}{2}\right)$ and $\frac{\partial P_{n}\left(x, \widetilde{\sigma}^{D}\right)}{x}<0$ for $x \in\left(\frac{1}{2}, 1-\sqrt{1 / 4+2 d-\sqrt{d}}\right]$.

Thus we have shown that $x=1 / 2$ is the best response of the advantaged candidate when the disadvantaged plays $\widetilde{\sigma}^{D}$ if $n$ is large enough. Combining the results described above we now have shown that a mixed strategy equilibrium exists for large enough values of $n$. Next we provide necessary conditions for existence of the equilibrium described above, that is, the minimal value of $n$ for which $\frac{\partial P_{n}\left(x, \widetilde{\sigma}^{D}\right)}{\partial x}>0$ for $x \in\left[\sqrt{1 / 4+2 d-\sqrt{d}}, \frac{1}{2}\right)$. Thus we characterize the necessary and sufficient conditions for $\widetilde{\sigma}^{A}=\frac{1}{2}$ and $\widetilde{\sigma}^{D}=\left(y=\frac{1}{2}-\sqrt{d}\right.$ with probability $\frac{1}{2}$ and $y=\frac{1}{2}+\sqrt{d}$ with probability $\frac{1}{2}$ ) to be equilibrium strategies.

Proposition $5 x=\frac{1}{2}$ is a global maximum of $P_{n}\left(x, \widetilde{\sigma}^{D}\right)$ if and only if $n \geq \frac{1}{4 d}$.

Given all the results developed above we can now state the main result in the following theorem.

Theorem The profile of mixed strategies $\widetilde{\sigma}^{A}=\frac{1}{2}$ and $\widetilde{\sigma}^{D}=\left(\frac{1}{2}-\sqrt{d} w \cdot p \cdot \frac{1}{2} ; \frac{1}{2}+\sqrt{d} w \cdot p \cdot \frac{1}{2}\right)$ constitutes a Nash equilibrium if and only if $n \geq \frac{1}{4 d}$.

The size of the non-policy advantage, $d$, determines the size of the electorate that is needed in order to guarantee the existence of the proposed equilibrium. The smaller the advantage, 
the larger the values of $n$ for which this equilibrium exists. In terms of our interpretation with the Beta distribution, for smaller values of the advantage we need the distribution of the ideal point of the median voter to be more concentrated around $1 / 2$ for this equilibrium to exist. As this value decreases we need the probability to be more concentrated around $1 / 2$. On the other hand, as the value of the advantage becomes smaller, that is, as the difference between the two candidates vanishes, the number of voters that are necessary to support the proposed strategies as an equilibrium increases, and at the same time the optimal strategy of the disadvantaged candidate moves closer to the advantage candidate's. That is both players' equilibrium strategies converge to the expected median voter's ideal point as candidate $A$ 's advantage shrinks to zero. On the contrary, as the advantage increases, candidates' differentiation increases and the equilibrium strategies of the disadvantaged candidate move away from $1 / 2$.

We also have that in equilibrium the probability with which the advantaged candidate wins is always larger than 1/2 and therefore larger than the disadvantaged's probability of winning. And as the value of the advantage increases the probability of winning of the advantaged candidate also increases. Observe that $P_{n}\left(\frac{1}{2}, \widetilde{\sigma}^{D}\right)=\frac{1}{2} P_{n}\left(\frac{1}{2}, 1 / 2-\sqrt{d}\right)+\frac{1}{2} P_{n}\left(\frac{1}{2}, 1 / 2+\sqrt{d}\right)=P_{n}\left(\frac{1}{2}, 1 / 2-\sqrt{d}\right)=$ $P_{n}\left(\frac{1}{2}, 1 / 2+\sqrt{d}\right)$. Therefore $P_{n}\left(\frac{1}{2}, \widetilde{\sigma}^{D}\right)=\sum_{k=\frac{n+1}{2}}^{n}\left(\begin{array}{l}n \\ k\end{array}\right) p\left(\frac{1}{2}, 1 / 2-\sqrt{d}\right)^{k}\left(1-p\left(\frac{1}{2}, 1 / 2-\sqrt{d}\right)\right)^{n-k}$. We know that $\sum_{k=\frac{n+1}{2}}^{n}\left(\begin{array}{l}n \\ k\end{array}\right) p\left(\frac{1}{2}, 1 / 2-\sqrt{d}\right)^{k}\left(1-p\left(\frac{1}{2}, 1 / 2-\sqrt{d}\right)\right)^{n-k}$ is increasing in $p\left(\frac{1}{2}, 1 / 2-\sqrt{d}\right)$. Since $p\left(\frac{1}{2}, 1 / 2-\sqrt{d}\right)$ is increasing in $d$ it follows that $P_{n}\left(\frac{1}{2}, \widetilde{\sigma}^{D}\right)$ is increasing in $d$ as well.

Finally, increasing the number of voters for a fixed value of the advantage leads to higher payoffs for the advantaged candidate. And when the number of voters tends to infinity, uncertainty disappears, and we have a pure strategy equilibrium with the advantaged candidate locating at the ideal point of the median voter winning for sure against any strategy of the disadvantaged candidate. 


\section{Concluding Remarks}

The main contribution of this paper is to characterize an equilibrium for the downsian model with an advantaged candidate when the policy space is continuous. We have shown that the features of this equilibrium are in line with those of the equilibria of similar models.

As in Aragones and Palfrey (2002) and Hummel (2010) in our equilibrium the advantaged candidate selects more centrist strategies than the disadvantaged candidate, and also the equilibrium strategies change with changes in the value of the advantage: they differentiate more whenever the advantage is larger. We also replicate their other equilibrium results: the advantaged candidate always wins with larger probability, and this probability increases with the value of the advantage.

Aragones and Palfrey (2002) also found that the level of uncertainty about the median voter affected the equilibrium strategies in such a way that greater uncertainty made the disadvantaged candidate move to the center, thus improving his equilibrium payoff. This implies that in their case the effect of the equality divergence is smaller when there is more uncertainty. In our case, the level of uncertainty does not affect the equilibrium strategies, but increasing uncertainty may lead to non existence of equilibrium. When that is not the case, we obtain as in Aragones and Palfrey (2004) that increasing uncertainty implies higher payoffs for the disadvantaged candidate. Notice that from the Condorcet Jury Theorem we have that $\sum_{k=\frac{n+1}{2}}^{n}\left(\begin{array}{l}n \\ k\end{array}\right) p\left(\frac{1}{2}, 1 / 2-\sqrt{d}\right)^{k}\left(1-p\left(\frac{1}{2}, 1 / 2-\sqrt{d}\right)\right)^{n-k}$ is increasing in $n$ whenever $p\left(\frac{1}{2}, 1 / 2-\sqrt{d}\right)>1 / 2$.

Finally, we can speculate about what happens when our equilibrium does not exist. We must have that in equilibrium candidate $A$ must be using a mixed strategy as well when $n<\frac{1}{4 d}$. Suppose otherwise. If $A$ is using a pure strategy then $D$ has a unique best response $\left(y=\frac{1}{2}-\sqrt{d}\right.$ if $x>\frac{1}{2}$ and $y=\frac{1}{2}+\sqrt{d}$ if $\left.x<\frac{1}{2}\right)$ unless $x=\frac{1}{2}$, in which case it has two best responses $y=\frac{1}{2}-\sqrt{d}$ and $y=\frac{1}{2}+\sqrt{d}$. Since a pure strategy equilibrium does not exist, in equilibrium we must be $x=\frac{1}{2}$. But then, from proposition 2 we know that $D$ 's best response is $\widetilde{\sigma}^{D}$. And in that case from proposition 3 we know that $A$ cannot use $x=\frac{1}{2}$ in equilibrium. Thus, $A$ must be using a mixed strategy as well. 


\section{REFERENCES}

Adams, James. 1999. Policy Divergence in Multicandidate Probabilistic Spatial Voting. Public Choice. 100: 103-22.

Ansolabehere, Stephen and James M. Snyder, Jr. 2000. Valence Politics and Equilibrium in Spatial Election Models. Public Choice. 103(June): 327-336.

Aragones, Enriqueta and Thomas R. Palfrey 2002. Mixed Equilibrium in a Downsian Model with a Favored Candidate. Journal of Economic Theory 103:131-161.

Aragones, Enriqueta and Thomas R. Palfrey 2004. The Effect of Candidate Quality on Electoral Equilibrium: An Experimental Study" American Political Science Review 98 (1): 77-90.

Aragones, Enriqueta and Thomas R. Palfrey 2005. "Electoral Competition Between Two Candidates of Different Quality: The Effects of Candidate Ideology and Private Information" in Social Choice and Strategic Decisions: Essays in Honor of Jeffrey S. Banks, Ed. David Austen-Smith and John Duggan, (2005) Berlin: Springer

Berger, Mark. M., Michael C. Munger, and Richard F. Potthoff. 2000. With Uncertainty, the Downsian Model Predicts Divergence. Journal of Theoretical Politics. 12 (April): 262-68.

Bernhardt, Daniel and Daniel Ingberman. 1985. Candidate Reputations and the Incumbency Effect. Journal of Public Economics. 27: 47-67.

Dasgupta, Partha and Eric Maskin. 1986. The Existence of Equilibrium in Discontinuous Economic Games I: Theory. Review of Economic Studies. 53: 1-26.

Glicksberg, I. L. 1952. A Further Generalization of the Kakutani Fixed Point Theorem with Application to Nash Equilibrium Points. Proceedings of the American Mathematical Society. 38: $170-74$.

Groseclose, Timothy 2001. A Model of Candidate Location When One Candidate Has a Valence Advantage. American Journal of Political Science 45 (October): 862-86. 
Hummel, Patrick 2010. On the nature of equilibria in a downsian model with candidate valence. Games and Economic Behavior 70 (2): 425-445.

Kiewiet, D. Roderick. 1983. Macroeconomics and Micropolitics. Chicago: University of Chicago Press.

Kiewiet, D. Roderick and Langche Zeng. 1993. "An Analysis of Congressional Career Decisions, 1947-86". American Political Science Review 87: 928-41

Kirstein, Roland and Georg v. Wagenheim. 2010. A Generalized Condorcet Jury Theorem with Two Independent Probabilities of Error. MAGKS Discussion Paper Series in Economics.

Londregan, John and Thomas Romer. 1993. Polarization, Incumbency, and the Personal Vote. in Political Economy: Institutions, Competition, and Representation (W.A. Barnett, M.J. Hinich, and N.J. Schofield eds.). Cambridge: Cambridge University Press. 355-77.

Sion, M. and P. Wolfe. 1957. On a Game Without a Value. Contributions to the Theory of Games, III. (Princeton: Annals of Mathematical Studies No. 39), 299-306.

Stokes, Donald E. 1963. Spatial Models of Party Competition. American Political Science Review. 57: $368-77$.

\section{Appendix}

\section{Proof of Proposition 1:}

Notice that if $d \geq \frac{1}{4}$ and the advantage selects a pure strategy $x=\frac{1}{2}$ then a voter with ideal point $x_{i}$ prefers to vote for the advantaged candidate if and only if $d-\left(x_{i}-\frac{1}{2}\right)^{2}>-\left(x_{i}-y\right)^{2}$ which is equivalent to $x_{i}(1-2 y)>-y^{2}-d+\frac{1}{4}$. For any strategy of the disadvantaged candidate $y<\frac{1}{2}$ we have that $x_{i}(1-2 y)>0>-y^{2}-d+\frac{1}{4}$ for all $x_{i}$, thus all voters prefer to vote for candidate $A$. Similarly, for any strategy of the disadvantaged candidate $y>\frac{1}{2}$ we have that all voters with $x_{i}<\frac{y^{2}+d-\frac{1}{4}}{2 y-1}$ prefer to vote for candidate $A$, but since $d \geq \frac{1}{4}$ implies $\frac{y^{2}+d-\frac{1}{4}}{2 y-1}>\frac{y^{2}}{2 y-1}>1$ for all $y \in[0,1]$, we have that all voters prefer to vote for candidate $A$. Thus, if $d \geq \frac{1}{4}$ there is 
a pure strategy equilibrium in which the advantaged candidate obtains the votes from all voters. Therefore, $A$ wins with probability one.

\section{Proof of proposition 2:}

Given that $x=\frac{1}{2}$, we search for a value of $y$ that maximizes the payoff function of the disadvantaged candidate, that is, the following expression.

$$
Q_{n}\left(\frac{1}{2}, y\right)=\sum_{k=\frac{n+1}{2}}^{n}\left(\begin{array}{l}
n \\
k
\end{array}\right) q\left(\frac{1}{2}, y\right)^{k}\left(1-q\left(\frac{1}{2}, y\right)\right)^{n-k}
$$

Kirstein and Wangenheim (2010) show that $\frac{\partial Q_{n}(x, y)}{\partial q}=n\left(\begin{array}{c}n-1 \\ \frac{n-1}{2}\end{array}\right)[q(x, y)(1-q(x, y))]^{\frac{n-1}{2}}>0$ for $x \geq \frac{1}{2}$. Thus we have that $Q_{n}\left(\frac{1}{2}, y\right)$ is strictly increasing in $q\left(\frac{1}{2}, y\right)$.

Therefore, in order to find a value of $y$ that maximizes $Q_{n}\left(\frac{1}{2}, y\right)$ it is enough to find the values $y<1 / 2$ that maximize $q\left(\frac{1}{2}, y\right)=\max \left\{0, \widehat{x}\left(\frac{1}{2}, y\right)\right\}=\max \left\{0, \frac{1}{4}+\frac{y}{2}-\frac{d}{1-2 y}\right\}$; and the values $y>1 / 2$ that maximize $q\left(\frac{1}{2}, y\right)=\max \{0,1-\widehat{x}(x, y)\}=\max \left\{0, \frac{3}{4}-\frac{y}{2}+\frac{d}{1-2 y}\right\}$

Notice that $1 / 2-\sqrt{d}=\arg \max \frac{1}{4}+\frac{y}{2}-\frac{d}{1-2 y}$, and $1 / 2-\sqrt{d} \in\left[0, \frac{1}{2}\right]$ when $d \in\left(0, \frac{1}{4}\right]$. Similarly, when $y>1 / 2$ we find that $1 / 2+\sqrt{d}=\arg \max \frac{3}{4}-\frac{y}{2}+\frac{d}{1-2 y}$ and $1 / 2+\sqrt{d} \in\left[\frac{1}{2}, 1\right]$ when $d \in\left(0, \frac{1}{4}\right]$.

Therefore, the proposed mixed strategy for candidate $D$ is a best response to $x=1 / 2$ for any $n>0$.

\section{Proof of proposition 3:}

If $n=1$ candidate $A$ 's probability of winning is given by

$$
P_{1}\left(x, \widetilde{\sigma}^{D}\right)=\frac{1}{2} p(x, 1 / 2-\sqrt{d})+\frac{1}{2} p(x, 1 / 2+\sqrt{d})
$$

We have seen that $P_{1}\left(x, \tilde{\sigma}^{D}\right)$ is increasing in $x \in\left[0, \sqrt{\frac{1}{4}+2 d-\sqrt{d}}\right)$ and that $P_{1}\left(x, \widetilde{\sigma}^{D}\right)$ is decreasing in $x \in\left(1-\sqrt{\frac{1}{4}+2 d-\sqrt{d}}, 1\right]$ and, therefore, the best response of $A$ must belong in $[\sqrt{1 / 4+2 d-\sqrt{d}}, 1-\sqrt{1 / 4+2 d-\sqrt{d}}]$. 
Observe that for all $d \in\left(0, \frac{1}{4}\right)$ we have that $1 / 2-\sqrt{d}<\sqrt{1 / 4+2 d-\sqrt{d}}<1 / 2<1-$ $\sqrt{1 / 4+2 d-\sqrt{d}}<1 / 2+\sqrt{d}$ and that if $n=1$ candidate $A$ 's probability of election $P_{1}\left(x, \tilde{\sigma}^{D}\right)=$ $\frac{1}{2} p(x, 1 / 2-\sqrt{d})+\frac{1}{2} p(x, 1 / 2+\sqrt{d})$ can also be written as

$$
\begin{aligned}
& P_{1}\left(x, \widetilde{\sigma}^{D}\right)=1 / 2\left(1-\frac{x+1 / 2-\sqrt{d}}{2}+\frac{d}{2(x-1 / 2+\sqrt{d})}\right)+1 / 2\left(\frac{x+1 / 2+\sqrt{d}}{2}-\frac{d}{2(x-1 / 2-\sqrt{d})}\right) \\
& \text { for } x \in[\sqrt{1 / 4+2 d-\sqrt{d}}, 1-\sqrt{1 / 4+2 d-\sqrt{d}}] .
\end{aligned}
$$

Thus, $\frac{\partial P_{1}\left(x, \widetilde{\sigma}^{D}\right)}{\partial x}=-\frac{d}{4(x-1 / 2+\sqrt{d})^{2}}+\frac{d}{4(x-1 / 2-\sqrt{d})^{2}}<0$ if and only if $(x-1 / 2+\sqrt{d})^{2}<(x-1 / 2-\sqrt{d})^{2}$. This implies that $P_{1}\left(x, \widetilde{\sigma}^{D}\right)$ is decreasing for $x \in[\sqrt{1 / 4+2 d-\sqrt{d}}, 1 / 2)$ and it is increasing for $x \in(1 / 2,1-\sqrt{1 / 4+2 d-\sqrt{d}}]$.

[Figure 2]

Therefore $P_{1}\left(x, \widetilde{\sigma}^{D}\right)$ is increasing for $x \in[0, \sqrt{1 / 4+2 d-\sqrt{d}}]$, decreasing for $x \in[\sqrt{1 / 4+2 d-\sqrt{d}}, 1 / 2)$ increasing for $x \in(1 / 2,1-\sqrt{1 / 4+2 d-\sqrt{d}}]$ and decreasing for $x \in[1-\sqrt{1 / 4+2 d-\sqrt{d}}, 1]$. This implies that when $n=1$ the optimal responses of candidate $A$ are either $x=\sqrt{1 / 4+2 d-\sqrt{d}}$ or $x=1-\sqrt{1 / 4+2 d-\sqrt{d}}$ but not $x=\frac{1}{2}$.

\section{Proof of proposition 4:}

Let's show that $\frac{\partial P_{n}\left(x, \widetilde{\sigma}^{D}\right)}{x}>0$ for $x \in\left[\sqrt{1 / 4+2 d-\sqrt{d}}, \frac{1}{2}\right)$. A similar analysis would prove that $\frac{\partial P_{n}\left(x, \tilde{\sigma}^{D}\right)}{x}<0$ for $x \in\left(\frac{1}{2}, 1-\sqrt{1 / 4+2 d-\sqrt{d}}\right]$.

Since $P_{n}\left(x, \widetilde{\sigma}^{D}\right)=\frac{1}{2} P_{n}(x, 1 / 2-\sqrt{d})+\frac{1}{2} P_{n}(x, 1 / 2+\sqrt{d})$ we have that

$$
\frac{\partial P_{n}\left(x, \widetilde{\sigma}^{D}\right)}{\partial x}=\frac{1}{2} \frac{\partial P_{n}(x, 1 / 2-\sqrt{d})}{\partial x}+\frac{1}{2} \frac{\partial P_{n}(x, 1 / 2+\sqrt{d})}{\partial x}
$$

where

$$
P_{n}(x, 1 / 2-\sqrt{d})=\sum_{k=\frac{n+1}{2}}^{n}\left(\begin{array}{l}
n \\
k
\end{array}\right) p(x, 1 / 2-\sqrt{d})^{k}(1-p(x, 1 / 2-\sqrt{d}))^{n-k}
$$


and

$$
P_{n}(x, 1 / 2+\sqrt{d})=\sum_{k=\frac{n+1}{2}}^{n}\left(\begin{array}{l}
n \\
k
\end{array}\right) p(x, 1 / 2+\sqrt{d})^{k}(1-p(x, 1 / 2+\sqrt{d}))^{n-k}
$$

We can compute the derivative of $P_{n}(x, 1 / 2-\sqrt{d})$ with respect to $p($.$) using the results in Kirstein$ and Wangenheim $(2010)$ and obtain $\frac{\partial P_{n}(x, 1 / 2-\sqrt{d})}{\partial p}=n\left(\begin{array}{c}n-1 \\ \frac{n-1}{2}\end{array}\right)[p(x, 1 / 2-\sqrt{d})(1-p(x, 1 / 2-\sqrt{d}))]^{\frac{n-1}{2}}$. Thus we have that the total derivative of $P_{n}(x, 1 / 2-\sqrt{d})$ with respect to $x$ by composing it with its partial derivative, that is, $\frac{\partial P_{n}(x, 1 / 2-\sqrt{d})}{\partial x}=\frac{\partial P_{n}(x, 1 / 2-\sqrt{d})}{\partial p} \frac{\partial p(x, 1 / 2-\sqrt{d})}{\partial x}$ which can be written as

$$
\frac{\partial P_{n}(x, 1 / 2-\sqrt{d})}{\partial x}=n\left(\begin{array}{c}
n-1 \\
\frac{n-1}{2}
\end{array}\right)[p(x, 1 / 2-\sqrt{d})(1-p(x, 1 / 2-\sqrt{d}))]^{\frac{n-1}{2}} \frac{\partial p(x, 1 / 2-\sqrt{d})}{\partial x}
$$

and similarly we have that

$$
\frac{\partial P_{n}(x, 1 / 2+\sqrt{d})}{\partial x}=n\left(\begin{array}{c}
n-1 \\
\frac{n-1}{2}
\end{array}\right)[p(x, 1 / 2+\sqrt{d})(1-p(x, 1 / 2+\sqrt{d}))]^{\frac{n-1}{2}} \frac{\partial p(x, 1 / 2+\sqrt{d})}{\partial x}
$$

Therefore,

$$
\begin{gathered}
\frac{\partial P_{n}\left(x, \widetilde{\sigma}^{D}\right)}{\partial x}=\frac{n}{2}\left(\begin{array}{l}
n-1 \\
\frac{n-1}{2}
\end{array}\right)\left[[p(x, 1 / 2-\sqrt{d})(1-p(x, 1 / 2-\sqrt{d}))]^{\frac{n-1}{2}} \frac{\partial p(x, 1 / 2-\sqrt{d})}{\partial x}+[p(x, 1 / 2+\sqrt{d})(1-\right. \\
\left.p(x, 1 / 2+\sqrt{d}))]^{\frac{n-1}{2}} \frac{\partial p(x, 1 / 2+\sqrt{d})}{\partial x}\right]
\end{gathered}
$$

and we need to prove that for large enough values of $n$ we have that

$$
\left.[p(x, 1 / 2-\sqrt{d})(1-p(x, 1 / 2-\sqrt{d}))]^{\frac{n-1}{2}} \frac{\partial p(x, 1 / 2-\sqrt{d})}{\partial x}+[p(x, 1 / 2+\sqrt{d})(1-p(x, 1 / 2+\sqrt{d}))]^{\frac{n-1}{2}} \frac{\partial p(x, 1 / 2+\sqrt{d})}{\partial x}\right]>
$$

0

whenever $x \in\left[\sqrt{1 / 4+2 d-\sqrt{d}}, \frac{1}{2}\right)$.

This holds if and only if

$$
\left[\frac{p(x, 1 / 2-\sqrt{d})(1-p(x, 1 / 2-\sqrt{d}))}{p(x, 1 / 2+\sqrt{d})(1-p(x, 1 / 2+\sqrt{d}))}\right]^{\frac{n-1}{2}} \frac{\partial p(x, 1 / 2-\sqrt{d})}{\partial x}+\frac{\partial p(x, 1 / 2+\sqrt{d})}{\partial x}>0
$$

First of all we will show that $\left[\frac{p(x, 1 / 2-\sqrt{d})(1-p(x, 1 / 2-\sqrt{d}))}{p(x, 1 / 2+\sqrt{d})(1-p(x, 1 / 2+\sqrt{d}))}\right]^{\frac{n-1}{2}}$ decreases with $n$ and it tends to zero as $n$ tends to infinite.

Notice that for $x \in\left[\sqrt{1 / 4+2 d-\sqrt{d}}, \frac{1}{2}\right)$ we have that $p(x, 1 / 2-\sqrt{d})>p(x, 1 / 2+\sqrt{d})>\frac{1}{2}$ 
which implies that $p(x, 1 / 2-\sqrt{d})(1-p(x, 1 / 2-\sqrt{d}))<p(x, 1 / 2+\sqrt{d})(1-p(x, 1 / 2+\sqrt{d}))$ always holds, since it does not depend on $n$.

Thus $\left[\frac{p(x, 1 / 2-\sqrt{d})(1-p(x, 1 / 2-\sqrt{d}))}{p(x, 1 / 2+\sqrt{d})(1-p(x, 1 / 2+\sqrt{d}))}\right]^{\frac{n-1}{2}}<1$ and $\lim _{n \rightarrow \infty}\left[\frac{p(x, 1 / 2-\sqrt{d})(1-p(x, 1 / 2-\sqrt{d}))}{p(x, 1 / 2+\sqrt{d})(1-p(x, 1 / 2+\sqrt{d}))}\right]^{\frac{n-1}{2}}=0$

Since we have that $\frac{\partial p(x, 1 / 2+\sqrt{d})}{\partial x}>0$ we also have that $\left[\frac{p(x, 1 / 2-\sqrt{d})(1-p(x, 1 / 2-\sqrt{d}))}{p(x, 1 / 2+\sqrt{d})(1-p(x, 1 / 2+\sqrt{d}))}\right]^{\frac{n-1}{2}} \frac{\partial p(x, 1 / 2-\sqrt{d})}{\partial x}+$ $\frac{\partial p(x, 1 / 2+\sqrt{d})}{\partial x}>0$ will hold for large values of $n$.

Similarly we could show that for $x \in(1 / 2,1-\sqrt{1 / 4+2 d-\sqrt{d}}]$ we have $\frac{\partial P_{n}\left(x, \tilde{\sigma}^{D}\right)}{x}<0$ which completes the proof of the proposition.

\section{Proof of proposition 5:}

From the last proposition we know that $\frac{\partial P_{n}\left(x, \widetilde{\sigma}^{D}\right)}{\partial x}>0$ for $x \in\left[\sqrt{1 / 4+2 d-\sqrt{d}}, \frac{1}{2}\right)$ if and only if

$\left[\frac{p(x, 1 / 2-\sqrt{d})(1-p(x, 1 / 2-\sqrt{d}))}{p(x, 1 / 2+\sqrt{d})(1-p(x, 1 / 2+\sqrt{d}))}\right]^{\frac{n-1}{2}} \frac{\partial p(x, 1 / 2-\sqrt{d})}{\partial x}+\frac{\partial p(x, 1 / 2+\sqrt{d})}{\partial x}>0$

which can also be written as

$n>2 \frac{\ln \left(-\frac{\frac{\partial p(x, 1 / 2+\sqrt{d})}{\partial x}}{\frac{\partial p(x, 1 / 2-\sqrt{d})}{\partial x}}\right)}{\ln \left[\frac{p(x, 1 / 2-\sqrt{d})(1-p(x, 1 / 2-\sqrt{d}))}{p(x, 1 / 2+\sqrt{d})(1-p(x, 1 / 2+\sqrt{d}))}\right]}+1$

because we have that $\frac{\partial p(x, 1 / 2-\sqrt{d})}{\partial x}<0$ and we also have that $\frac{p(x, 1 / 2-\sqrt{d})(1-p(x, 1 / 2-\sqrt{d}))}{p(x, 1 / 2+\sqrt{d})(1-p(x, 1 / 2+\sqrt{d}))}<1$ implies $\ln \left[\frac{p(x, 1 / 2-\sqrt{d})(1-p(x, 1 / 2-\sqrt{d}))}{p(x, 1 / 2+\sqrt{d})(1-p(x, 1 / 2+\sqrt{d}))}\right]<0$.

For $x=1 / 2$ to be a local maximum we need to have $\frac{\partial P_{n}\left(x, \widetilde{\sigma}^{D}\right)}{\partial x} \geq 0$ for $x=1 / 2-\varepsilon$ where $\varepsilon>0$ and $\varepsilon \rightarrow 0$.

Thus we have to compute $\lim _{x \rightarrow 1 / 2} 2 \frac{\ln \left(-\frac{\frac{\partial p(x, 1 / 2+\sqrt{d})}{\partial x}}{\frac{\partial p(x, 1 / 2-\sqrt{d})}{\partial x}}\right)}{\ln \left[\frac{p(x, 1 / 2-\sqrt{d})(1-p(x, 1 / 2-\sqrt{d}))}{p(x, 1 / 2+\sqrt{d})(1-p(x, 1 / 2+\sqrt{d}))}\right]}+1$ 
Notice that when $x$ approaches $\frac{1}{2}$ we have that $-\frac{\frac{\partial p(x, 1 / 2+\sqrt{d})}{\partial x}}{\frac{\partial p(x, 1 / 2-\sqrt{d})}{\partial x}} \rightarrow 1$ and $\frac{p(x, 1 / 2-\sqrt{d})(1-p(x, 1 / 2-\sqrt{d}))}{p(x, 1 / 2+\sqrt{d})(1-p(x, 1 / 2+\sqrt{d}))} \rightarrow$ 1, thus we may apply l'Hopital's rule and we obtain that

$$
\lim _{x \rightarrow 1 / 2} \frac{\ln \left(-\frac{\frac{\partial p(x, 1 / 2+\sqrt{d})}{\partial x}}{\frac{\partial p(x, 1 / 2-\sqrt{d})}{\partial x}}\right)}{\ln \left[\frac{p(x, 1 / 2-\sqrt{d}(1-p(x, 1 / 2-\sqrt{d}))}{p(x, 1 / 2+\sqrt{d})(1-p(x, 1 / 2+\sqrt{d})}\right]}=\lim _{x \rightarrow 1 / 2} \frac{\left(-\frac{\frac{\partial p(x, 1 / 2-\sqrt{d})}{\partial x}}{\frac{\partial p(x, 1 / 2+\sqrt{d})}{\partial x}}\right) \frac{\partial\left(-\frac{\frac{\partial p(x, 1 / 2+\sqrt{d})}{\partial x}}{\frac{\partial p(x, 1 / 2-\sqrt{d})}{\partial x}}\right)}{\partial x}}{\frac{p(x, 1 / 2+\sqrt{d})(1-p(x, 1 / 2+\sqrt{d}))}{p(x, 1 / 2-\sqrt{d})(1-p(x, 1 / 2-\sqrt{d}))} \frac{\partial\left[\frac{p(x, 1 / 2-\sqrt{d})(1-p(x, 1 / 2-\sqrt{d}))}{p(x, 1 / 2+\sqrt{d})(1-p(x, 1 / 2+\sqrt{d}))}\right]}{\partial x}}
$$

Observe that since for $x \in(1 / 2-\sqrt{d}, 1 / 2+\sqrt{d})$ we have that

$p\left(x, \frac{1}{2}-\sqrt{d}\right)=1-\frac{x+\frac{1}{2}-\sqrt{d}}{2}-\frac{d}{2\left(\frac{1}{2}-\sqrt{d}-x\right)}$ and $p\left(x, \frac{1}{2}+\sqrt{d}\right)=\frac{x+\frac{1}{2}+\sqrt{d}}{2}+\frac{d}{2\left(\frac{1}{2}+\sqrt{d}-x\right)}$

then

$\frac{\partial p\left(x, \frac{1}{2}-\sqrt{d}\right)}{\partial x}=-\frac{1}{2}-\frac{d}{2\left(\frac{1}{2}-\sqrt{d}-x\right)^{2}}$ and $\frac{\partial p\left(x, \frac{1}{2}+\sqrt{d}\right)}{\partial x}=\frac{1}{2}+\frac{d}{2\left(\frac{1}{2}+\sqrt{d}-x\right)^{2}}$

which implies that

$\left(-\frac{\frac{\partial p(x, 1 / 2-\sqrt{d})}{\partial x}}{\frac{\partial p(x, 1 / 2+\sqrt{d})}{\partial x}}\right)=\frac{1+\frac{d}{\left(\frac{1}{2}-\sqrt{d}-x\right)^{2}}}{1+\frac{d}{\left(\frac{1}{2}+\sqrt{d}-x\right)^{2}}} \rightarrow 1$

and

$\frac{\partial\left(-\frac{p(x, 1 / 2+\sqrt{d})(1-p(x, 1 / 2+\sqrt{d}))}{\frac{\partial p(x, 1 / 2-\sqrt{d})}{\partial x}}\right)}{\partial x}=\frac{\frac{2 d}{\left(\frac{1}{2}+\sqrt{d}-x\right)^{3}}-\frac{2 d}{\left(\frac{1}{2}-\sqrt{d}-x\right)^{3}}+\frac{2 d^{2}}{\left(\frac{1}{2}+\sqrt{d}-x\right)^{2}\left(\frac{1}{2}-\sqrt{d}-x\right)^{2}}\left(\frac{1}{\left(\frac{1}{2}+\sqrt{d}-x\right)}-\frac{1}{\left(\frac{1}{2}-\sqrt{d}-x\right)}\right)}{\left(1+\frac{d}{\left(\frac{1}{2}-\sqrt{d}-x\right)^{2}}\right)^{2}} \rightarrow \frac{2}{\sqrt{d}}$

Similarly we find that

$\frac{p(x, 1 / 2+\sqrt{d})(1-p(x, 1 / 2+\sqrt{d}))}{p(x, 1 / 2-\sqrt{d})(1-p(x, 1 / 2-\sqrt{d}))} \rightarrow \frac{\frac{1}{4}-d}{\frac{1}{4}-d}=1$

and

$\frac{\partial\left[\frac{p(x, 1 / 2-\sqrt{d})(1-p(x, 1 / 2-\sqrt{d}))}{p(x, 1 / 2+\sqrt{d})(1-p(x, 1 / 2+\sqrt{d}))}\right]}{\partial x}=$

$=\frac{(1-2 p(x, 1 / 2-\sqrt{d})) \frac{\partial p(x, 1 / 2-\sqrt{d})}{\partial x}}{p(x, 1 / 2+\sqrt{d})(1-p(x, 1 / 2+\sqrt{d}))}-\frac{p(x, 1 / 2+\sqrt{d})(1-2 p(x, 1 / 2+\sqrt{d})) \frac{\partial p(x, 1 / 2+\sqrt{d})}{\partial x} p(x, 1 / 2-\sqrt{d})(1-p(x, 1 / 2-\sqrt{d}))}{p(x, 1 / 2+\sqrt{d})^{2}(1-p(x, 1 / 2+\sqrt{d}))^{2}} \rightarrow \frac{4 \sqrt{d}}{\frac{1}{4}-d}$

because we have that 
$\frac{\partial p(x, 1 / 2-\sqrt{d})}{\partial x} \rightarrow-1 ; \frac{\partial p(x, 1 / 2+\sqrt{d})}{\partial x} \rightarrow+1$

and

$1-2 p(x, 1 / 2-\sqrt{d}) \rightarrow-2 \sqrt{d} ; 1-2 p(x, 1 / 2+\sqrt{d}) \rightarrow-2 \sqrt{d}$

Therefore, we obtain that

$\lim _{x \rightarrow 1 / 2} 2 \frac{\ln \left(-\frac{\frac{\partial p(x, 1 / 2+\sqrt{d})}{\partial x}}{\frac{\partial p(x, 1 / 2-\sqrt{d})}{\partial x}}\right)}{\ln \left[\frac{p(x, 1 / 2-\sqrt{d})(1-p(x, 1 / 2-\sqrt{d}))}{p(x, 1 / 2+\sqrt{d})(1-p(x, 1 / 2+\sqrt{d}))}\right]}+1=2\left(\frac{1-4 d}{8 d}\right)+1=\frac{1}{4 d}$

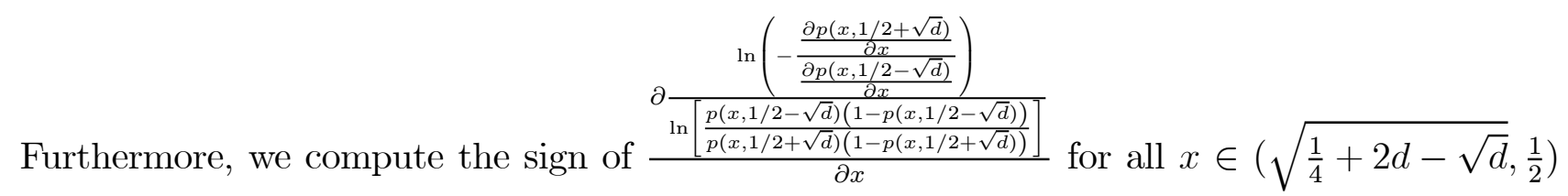
and for all $d \in\left(0, \frac{1}{4}\right)$ using Mathematica and we get that it is positive (see figure 7 ).

This implies that if $n>\frac{\ln \left(-\frac{\frac{\partial p(x, 1 / 2+\sqrt{d})}{\partial x}}{\frac{\partial p(x, 1 / 2-\sqrt{d})}{\partial x}}\right)}{\ln \left[\frac{p(x, 1 / 2-\sqrt{d})(1-p(x, 1 / 2-\sqrt{d}))}{p(x, 1 / 2+\sqrt{d})(1-p(x, 1 / 2+\sqrt{d})}\right]}+1$ for $x \rightarrow \frac{1}{2}$ then the inequality should hold for all $x \in\left(\sqrt{\frac{1}{4}+2 d-\sqrt{d}}, \frac{1}{2}\right)$ as well.

Thus, $x=\frac{1}{2}$ is a global maximum if and only if $n \geq \frac{1}{4 d}$. 


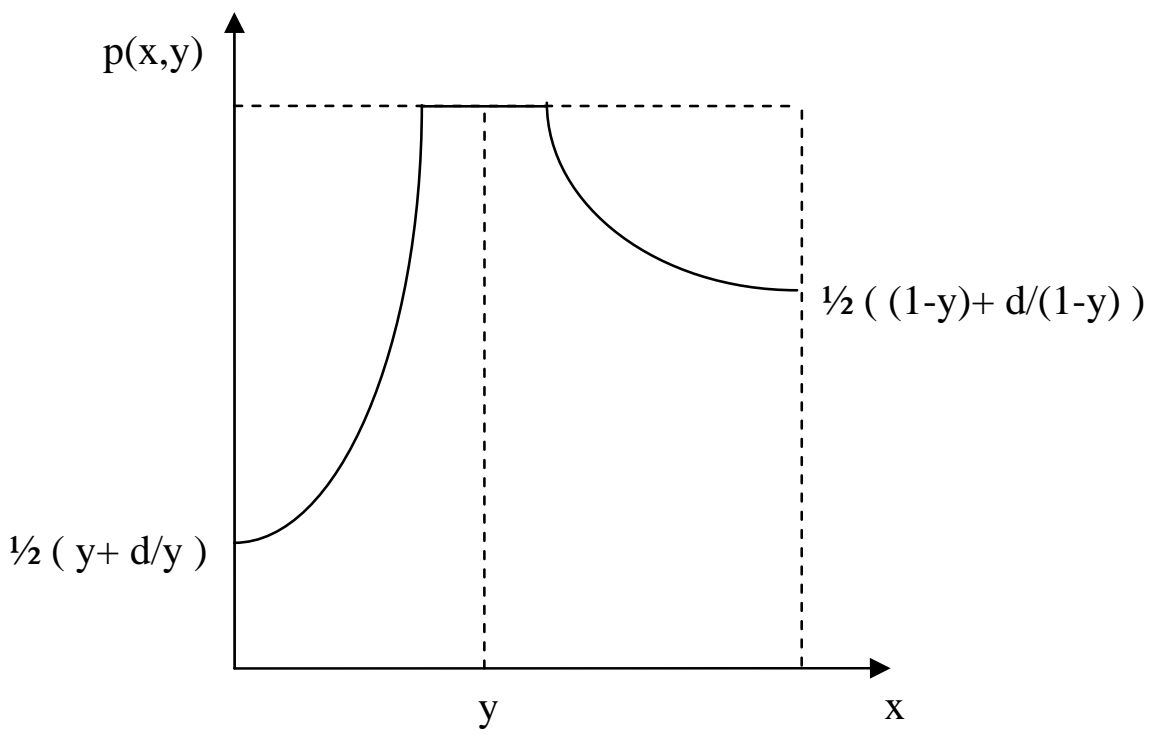

Figure 1: The probability that a voter votes for the advantaged candidate, $\mathrm{p}(\mathrm{x}, \mathrm{y})$, as a function of the advantaged candidate's policy choice $(\mathrm{x})$ given a policy choice of the disadvantaged candidate (y). 


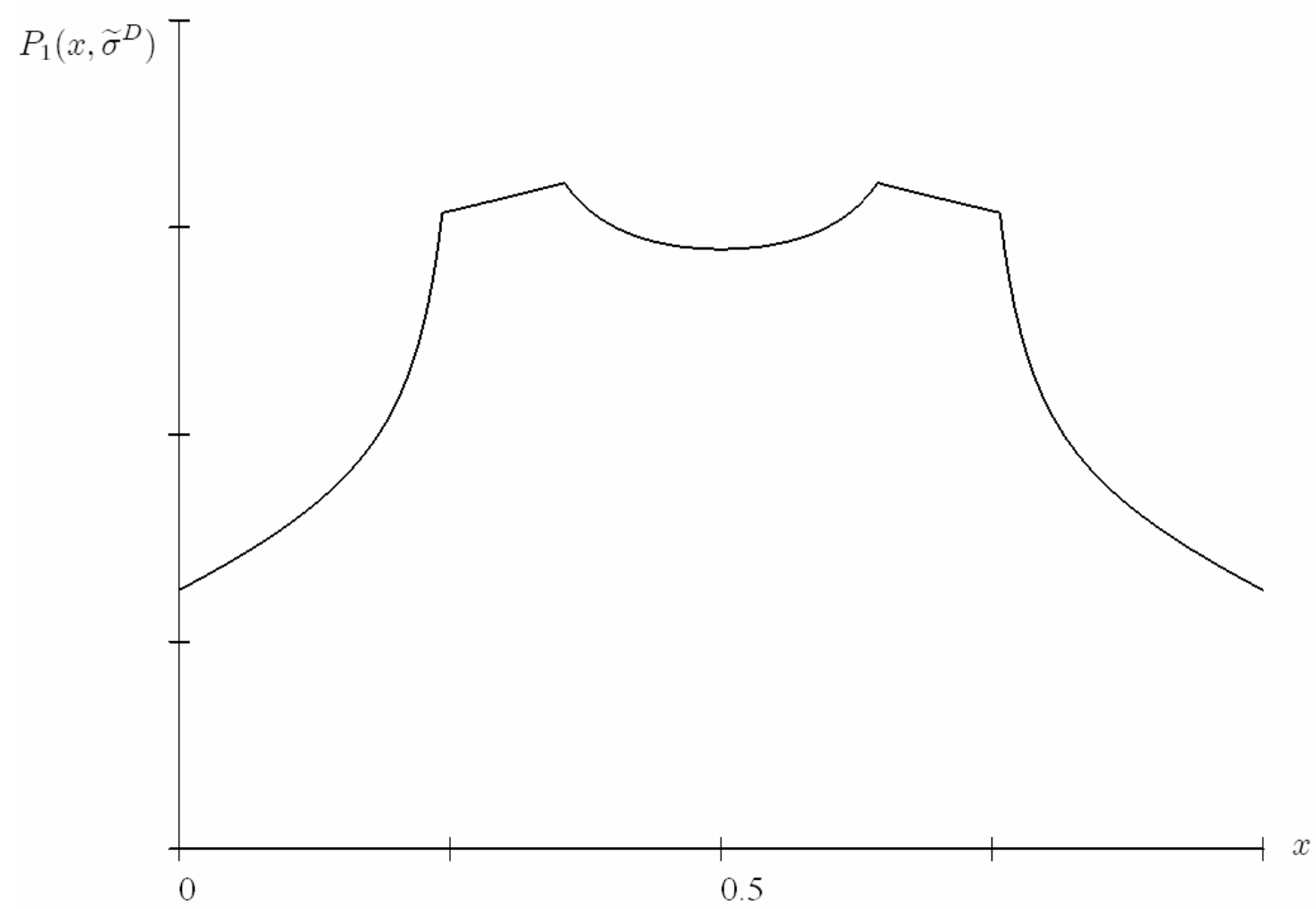

Figure 2: The probability that candidate $\mathrm{A}, \mathrm{P}_{1}\left(\mathrm{x}, \sigma^{\mathrm{D}}\right)$, as a function of the advantaged candidate's policy choice $(\mathrm{x})$ given the best response of the disadvantaged candidate $\left(\sigma^{\mathrm{D}}\right)$ when $\mathrm{n}=1$ and $\mathrm{d}=0.05$. 


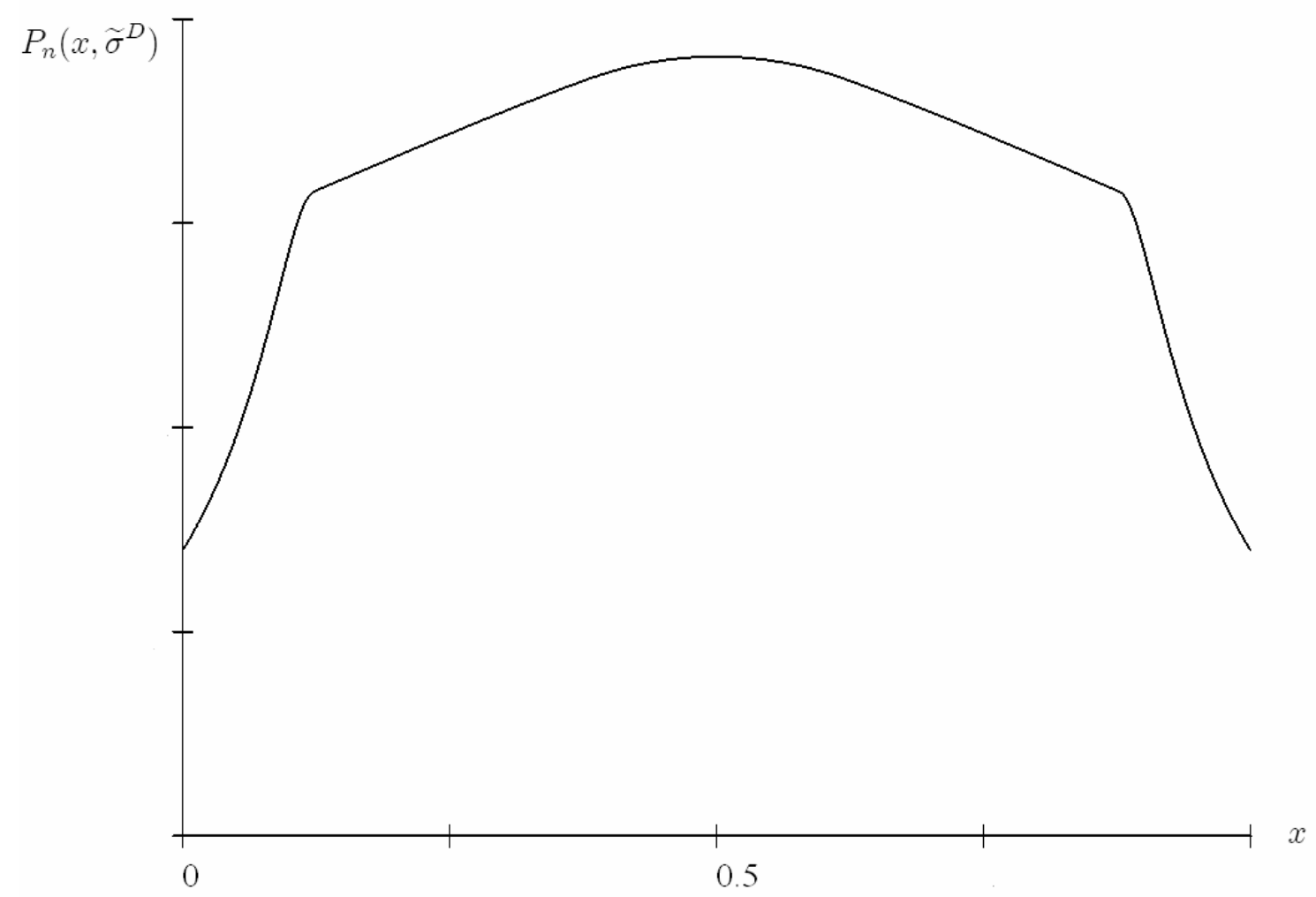

Figure 3: The probability that candidate $A$ wins, $P_{n}\left(x, \sigma^{D}\right)$, as a function of the advantagedcandidate's policy choice $(\mathrm{x})$ given the best response of the disadvantaged candidate $\left(\sigma^{\mathrm{D}}\right)$ when $n=5$ and $d=0.1$, thus $n>1 / 4 d$ 


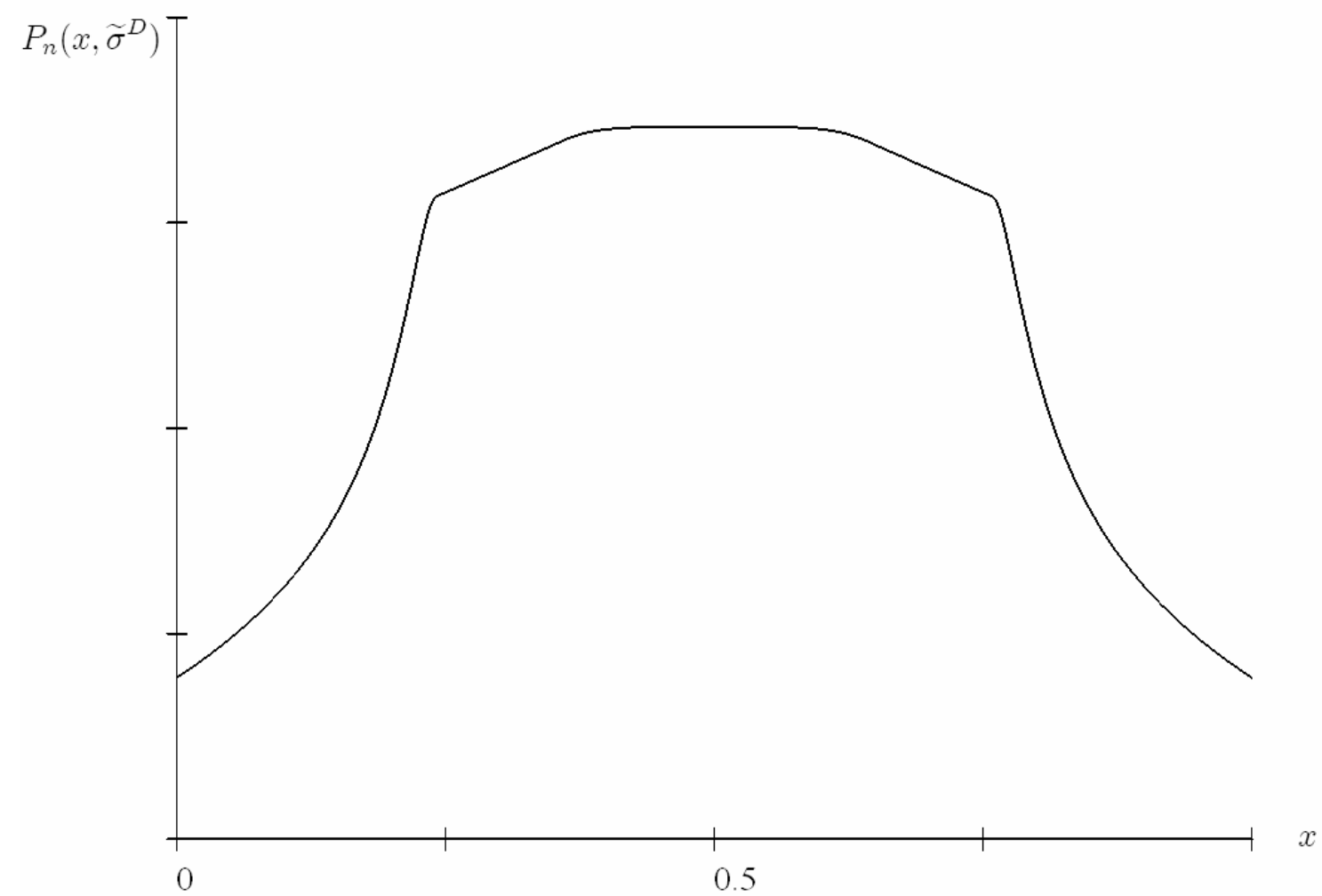

Figure 4: The probability that candidate A wins, $P_{n}\left(x, \sigma^{D}\right)$, as a function of the advantaged candidate's policy choice (x) given the best response of the disadvantaged candidate $\left(\sigma^{\mathrm{D}}\right)$ when $\mathrm{n}=5$ and $\mathrm{d}=0.05$ thus $\mathrm{n}=1 / 4 \mathrm{~d}$. 


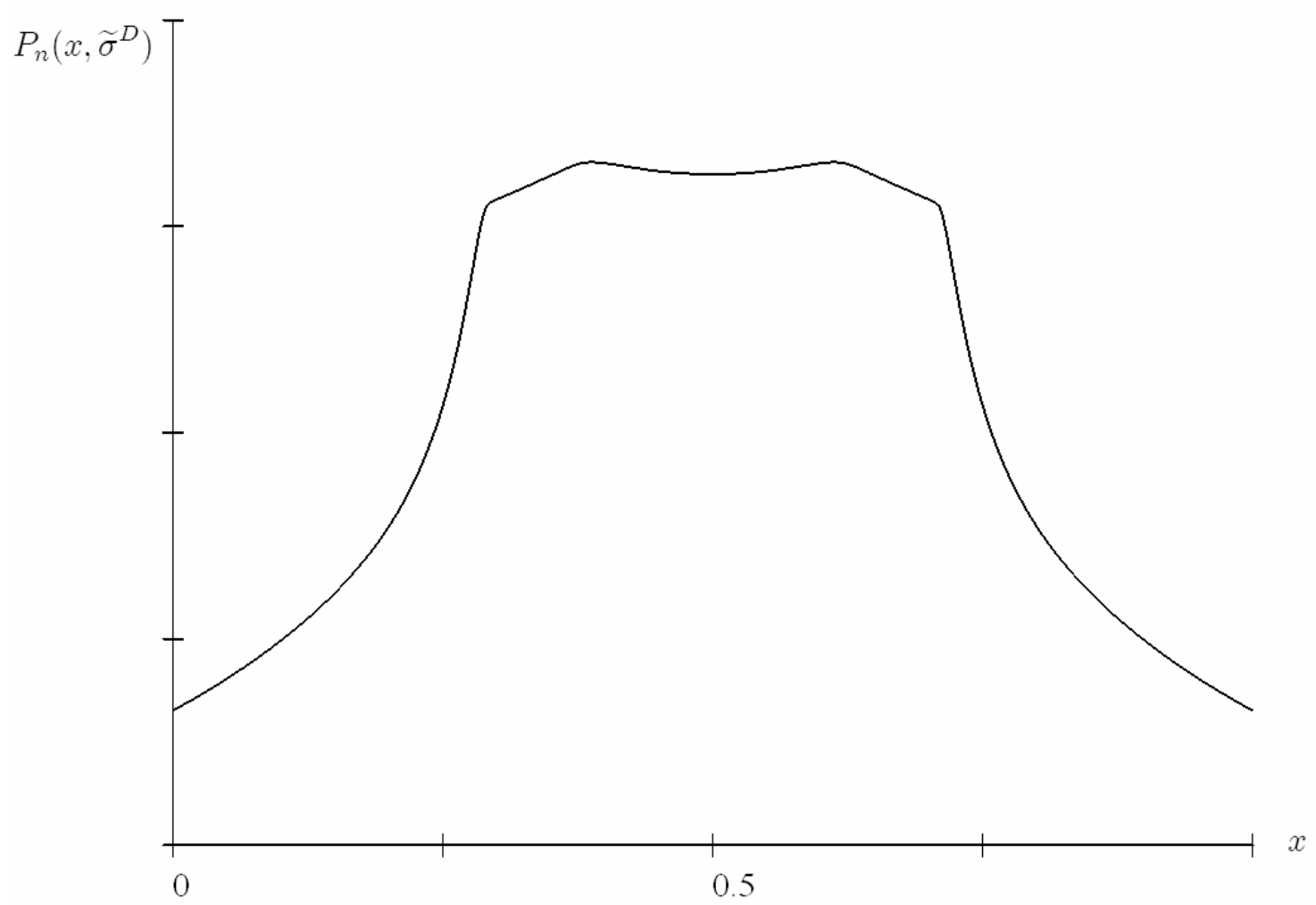

Figure 5: The probability that candidate A wins, $\mathrm{P}_{\mathrm{n}}\left(\mathrm{x}, \sigma^{\mathrm{D}}\right)$, as a function of the advantaged candidate's policy choice $(\mathrm{x})$ given the best response of the disadvantaged candidate $\left(\sigma^{\mathrm{D}}\right)$ when $\mathrm{n}=5$ and $\mathrm{d}=0.03$ thus $\mathrm{n}<1 / 4 \mathrm{~d}$. 


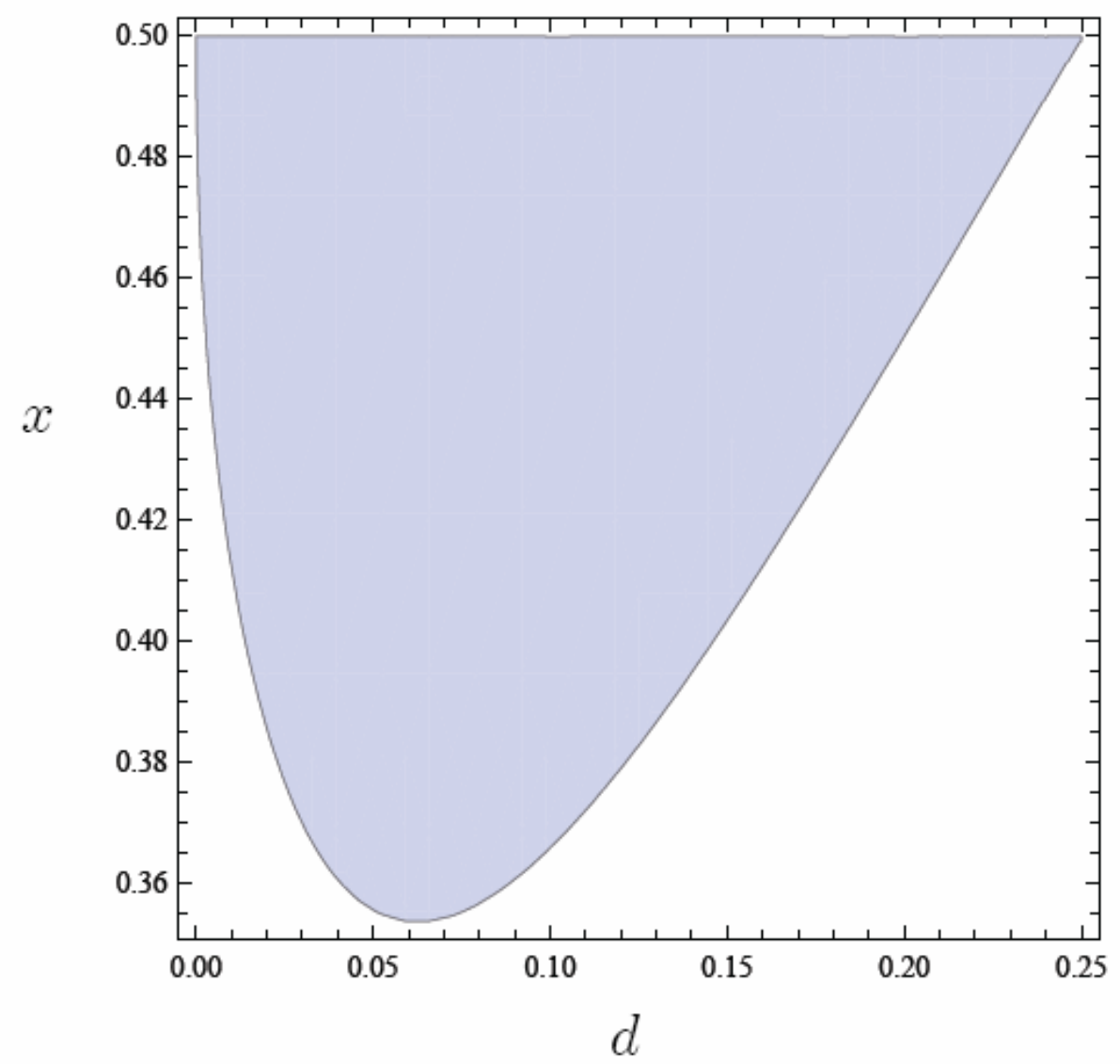

Figure 6: Critical values of the payoff function of the advantaged candidate as a function of the advantage size $d$. The shaded area represents $x \in\left(\sqrt{\frac{1}{4}+2 d-\sqrt{d}}, \frac{1}{2}\right)$. 


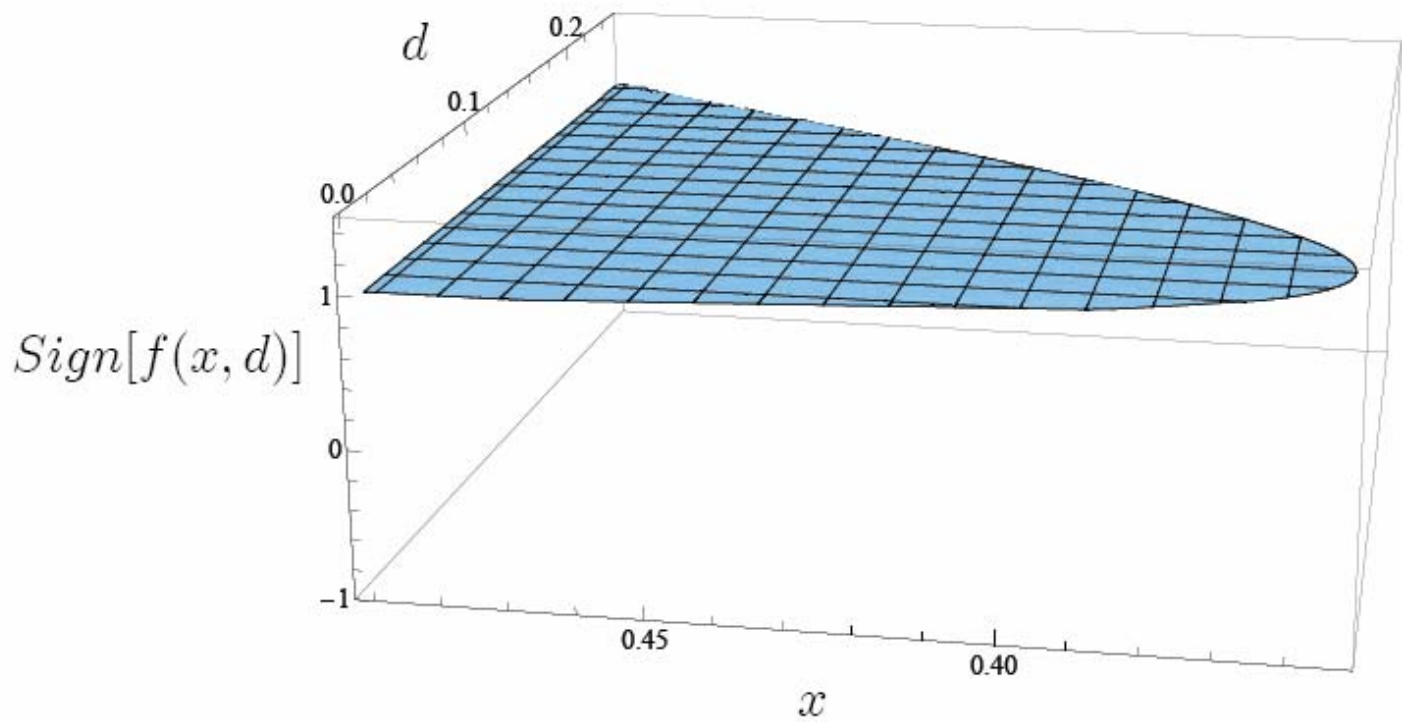

Figure 7: The sign of the derivative of the advantage payoff function for the critical values of the payoff function of the advantaged candidate computed by Mathematica is always positive. 